\title{
Properties of hyperbolic generalized Pell numbers
}

\author{
Yüksel Soykan $^{1}$ and Melih Göcen ${ }^{2}$ \\ ${ }^{1}$ Department of Mathematics, Zonguldak Bülent Ecevit University \\ 67100, Zonguldak, Turkey \\ e-mail: yuksel_soykan@hotmail.com \\ ${ }^{2}$ Department of Mathematics, Zonguldak Bülent Ecevit University \\ 67100, Zonguldak, Turkey \\ e-mail: gocenmehotmail.com
}

Received: 9 June 2020

Revised: 31 October 2020

Accepted: 11 November 2020

\begin{abstract}
In this paper, we introduce the generalized hyperbolic Pell numbers over the bidimensional Clifford algebra of hyperbolic numbers. As special cases, we deal with hyperbolic Pell and hyperbolic Pell-Lucas numbers. We present Binet's formulas, generating functions and the summation formulas for these numbers. Moreover, we give Catalan's, Cassini's, d'Ocagne's, Gelin-Cesàro's, Melham's identities and present matrices related to these sequences.
\end{abstract}

Keywords: Pell numbers, Pell-Lucas numbers, Hyperbolic numbers, Hyperbolic Pell numbers, Cassini identity.

2010 Mathematics Subject Classification: 11B39, 11B83.

\section{Introduction}

There are some extensions (generalizations) of real numbers into real algebras of dimension 2 which are the followings: complex numbers,

$$
\mathbb{C}=\left\{z=a+i b: a, b \in \mathbb{R}, i^{2}=-1\right\},
$$

hyperbolic (double, split-complex) numbers [28],

$$
\mathbb{H}=\left\{h=a+h b: a, b \in \mathbb{R}, h^{2}=1\right\},
$$

and dual numbers [10],

$$
\mathbb{D}=\left\{d=a+\varepsilon b: a, b \in \mathbb{R}, \varepsilon^{2}=0\right\} .
$$


In fact, each possible system can be reduced to one of the above and there exist essentially three possible ways to generalize real numbers into real algebras of dimension 2 (see, for example, [16] for details).

There are also other extensions (generalizations) of real numbers into real algebras of higher dimension. The hypercomplex numbers systems, [16], are extensions of real numbers. Some commutative examples of hypercomplex number systems are complex numbers, hyperbolic numbers, [28], and dual numbers, [10]. Some non-commutative examples of hypercomplex number systems are quaternions [12], octonions [2] and sedenions [30]. The algebras $\mathbb{C}$ (complex numbers), $\mathbb{H}_{\mathbb{Q}}$ (quaternions), $\mathbb{O}$ (octonions) and $\mathbb{S}$ (sedenions) are real algebras obtained from the real numbers $\mathbb{R}$ by a doubling procedure called the Cayley-Dickson Process. This doubling process can be extended beyond the sedenions to form what are known as the $2^{n}$-ions (see for example $[4,14,24])$.

Quaternions were invented by Irish mathematician W. R. Hamilton (1805-1865) [12] as an extension to the complex numbers. Hyperbolic numbers with complex coefficients are introduced by J. Cockle in 1848, [6]. H. H. Cheng and S. Thompson [5] introduced dual numbers with complex coefficients and called complex dual numbers. Akar, Yüce and Şahin [1] introduced dual hyperbolic numbers.

Here we use the set of hyperbolic numbers. The set of hyperbolic numbers $\mathbb{H}$ can be described as

$$
\mathbb{H}=\left\{z=x+h y \mid h \notin \mathbb{R}, h^{2}=1, x, y \in \mathbb{R}\right\} .
$$

The hyperbolic ring $\mathbb{H}$ is a bidimensional Clifford algebra, see [20] for details. Hyperbolic numbers has been called in the mathematical literature with different names: Lorentz numbers, double numbers, duplex numbers, split complex numbers and perplex numbers. Hyperbolic numbers are useful for measuring distances in the Lorentz space-time plane (see Sobczyk [28]). For more information on hyperbolic numbers, see also [15, 22, 25, 29].

Addition, substraction and multiplication of any two hyperbolic numbers $z_{1}$ and $z_{2}$ are defined by

$$
\begin{aligned}
& z_{1} \pm z_{2}=\left(x_{1}+h y_{1}\right) \pm\left(x_{2}+h y_{2}\right)=\left(x_{1} \pm x_{2}\right)+h\left(y_{1} \pm y_{2}\right) \\
& z_{1} \times z_{2}=\left(x_{1}+h y_{1}\right) \times\left(x_{2}+h y_{2}\right)=x_{1} x_{2}+y_{1} y_{2}+h\left(x_{1} y_{2}+y_{1} x_{2}\right) .
\end{aligned}
$$

and the division of two hyperbolic numbers are given by

$$
\frac{z_{1}}{z_{2}}=\frac{x_{1}+h y_{1}}{x_{2}+h y_{2}}=\frac{\left(x_{1}+h y_{1}\right)\left(x_{2}-h y_{2}\right)}{\left(x_{2}+h y_{2}\right)\left(x_{2}-h y_{2}\right)}=\frac{x_{1} x_{2}+y_{1} y_{2}}{x_{2}^{2}-y_{2}^{2}}+h \frac{x_{1} y_{2}+y_{1} x_{2}}{x_{2}^{2}-y_{2}^{2}} .
$$

It is easy to see that this algebra of hyperbolic numbers is commutative and contains zero divisors. The hyperbolic conjugation of $z=x+h y$ is defined by

$$
\bar{z}=z^{\dagger}=x-h y .
$$

Note that $\overline{\bar{z}}=z$. Note also that for any hyperbolic numbers $z_{1}, z_{2}, z$ we have

$$
\begin{aligned}
\overline{z_{1}+z_{2}} & =\overline{z_{1}}+\overline{z_{2}}, \\
\overline{z_{1} \times z_{2}} & =\overline{z_{1}} \times \overline{z_{2}}, \\
\|z\|^{2} & =z \times \bar{z}=x^{2}-y^{2} .
\end{aligned}
$$

Now let us recall the definition of generalized Pell numbers. 
A generalized Pell sequence $\left\{V_{n}\right\}_{n \geq 0}=\left\{V_{n}\left(V_{0}, V_{1}\right)\right\}_{n \geq 0}$ is defined by the second-order recurrence relations

$$
V_{n}=2 V_{n-1}+V_{n-2} ; \quad V_{0}=a, V_{1}=b, \quad(n \geq 2)
$$

with the initial values $V_{0}, V_{1}$ not all being zero. The sequence $\left\{V_{n}\right\}_{n \geq 0}$ can be extended to negative subscripts by defining

$$
V_{-n}=-2 V_{-(n-1)}+V_{-(n-2)}
$$

for $n=1,2,3, \ldots$. Therefore, recurrence (1.1) holds for all integer $n$.

The first few generalized Pell numbers with positive subscript and negative subscript are given in the following Table 1.

\begin{tabular}{|c||c|c|}
\hline$n$ & $V_{n}$ & $V_{-n}$ \\
\hline \hline 0 & $V_{0}$ & \\
1 & $V_{1}$ & $-2 V_{0}+V_{1}$ \\
2 & $V_{0}+2 V_{1}$ & $5 V_{0}-2 V_{1}$ \\
3 & $2 V_{0}+5 V_{1}$ & $-12 V_{0}+5 V_{1}$ \\
4 & $5 V_{0}+12 V_{1}$ & $29 V_{0}-12 V_{1}$ \\
5 & $12 V_{0}+29 V_{1}$ & $-70 V_{0}+29 V_{1}$ \\
\hline
\end{tabular}

Table 1. A few generalized Pell numbers

If we set $V_{0}=0, V_{1}=1$ then $\left\{V_{n}\right\}$ is the well-known Pell sequence and if we set $V_{0}=2, V_{1}=2$ then $\left\{V_{n}\right\}$ is the well-known Pell-Lucas sequence. In other words, Pell sequence $\left\{P_{n}\right\}_{n \geq 0}$ (OEIS: A000129, [27]) and Pell-Lucas sequence $\left\{Q_{n}\right\}_{n \geq 0}$ (OEIS: A002203, [27]) are defined by the second-order recurrence relations

$$
P_{n}=2 P_{n-1}+P_{n-2}, \quad P_{0}=0, P_{1}=1
$$

and

$$
Q_{n}=2 Q_{n-1}+Q_{n-2}, \quad Q_{0}=2, Q_{1}=2 .
$$

The sequences $\left\{P_{n}\right\}_{n \geq 0}$ and $\left\{Q_{n}\right\}_{n \geq 0}$ can be extended to negative subscripts by defining

$$
P_{-n}=-2 P_{-(n-1)}+P_{-(n-2)}
$$

and

$$
Q_{-n}=-2 Q_{-(n-1)}+Q_{-(n-2)}
$$

for $n=1,2,3, \ldots$ respectively. Therefore, recurrences (1.2) and (1.3) hold for all integer $n$.

Pell sequence has been studied by many authors and more detail can be found in the extensive literature dedicated to these sequences, see for example, $[3,7,9,11,13,17,21,23]$. For higher order Pell sequences, see [18, 19,32-34].

We can list some important properties of generalized Pell numbers that are needed. 
- Binet formula of generalized Pell sequence can be calculated using its characteristic equation which is given as $t^{2}-2 t-1=0$. The roots of characteristic equation are $\alpha=1+\sqrt{2}, \quad \beta=1-\sqrt{2}$ and the roots satisfy the following $\alpha+\beta=2, \alpha \beta=-1$, $\alpha-\beta=2 \sqrt{2}$. Using these roots and the recurrence relation, Binet formula can be given as

$$
V_{n}=\frac{A \alpha^{n}-B \beta^{n}}{\alpha-\beta},
$$

where $A=V_{1}-V_{0} \beta$ and $B=V_{1}-V_{0} \alpha$.

- Binet formula of Pell and Pell-Lucas sequences are $P_{n}=\frac{\alpha^{n}-\beta^{n}}{\alpha-\beta}$ and $Q_{n}=\alpha^{n}+\beta^{n}$, respectively.

- The generating function for generalized Pell numbers is:

$$
g(t)=\frac{W_{0}+\left(W_{1}-2 W_{0}\right) t}{1-2 t-t^{2}}
$$

- The Cassini identity for generalized Pell numbers is:

$$
V_{n+1} V_{n-1}-V_{n}^{2}=\left(2 V_{0} V_{1}-V_{1}^{2}-V_{0}^{2}\right) .
$$

- The generalized Pell sequence has the following properties:

$$
\begin{aligned}
& A \alpha^{n}=\alpha V_{n}+V_{n-1}, \\
& B \beta^{n}=\beta V_{n}+V_{n-1} .
\end{aligned}
$$

In this paper, we define the hyperbolic generalized Pell numbers in the next section and give some properties of them.

\section{Hyperbolic generalized Pell numbers and their generating functions and Binet's formulas}

In this section, we define hyperbolic generalized Pell numbers and present generating functions and Binet formulas for them.

In [36], the author defined hyperbolic Fibonacci numbers and Dikmen [8] defined hyperbolic Jacobsthal numbers. Soykan [35], defined hyperbolic generalized Fibonacci numbers.

We now define hyperbolic generalized Pell numbers over $\mathbb{H}$. The $n$-th hyperbolic generalized Pell number is

$$
\widetilde{V}_{n}=V_{n}+h V_{n+1}
$$

with initial conditions $\widetilde{V}_{0}=V_{0}+h V_{1}, \widetilde{V}_{1}=V_{1}+h\left(V_{0}+2 V_{1}\right)$ where $h^{2}=1$. As special cases, the $n$-th hyperbolic Pell numbers and the $n$-th hyperbolic Pell-Lucas numbers are given as $\widetilde{P}_{n}=P_{n}+h P_{n+1}$ and $\widetilde{Q}_{n}=Q_{n}+h Q_{n+1}$, respectively. It can be easily shown that

$$
\widetilde{V}_{n}=2 \widetilde{V}_{n-1}+\widetilde{V}_{n-2}
$$

The sequence $\left\{\widetilde{V}_{n}\right\}_{n \geq 0}$ can be extended to negative subscripts by defining

$$
\widetilde{V}_{-n}=-2 \widetilde{V}_{-(n-1)}+\widetilde{V}_{-(n-2)}
$$

for $n=1,2,3, \ldots$, respectively. Therefore, recurrence (2.2) holds for all integers $n$. 
Note that $\widetilde{V}_{n} h=V_{n+1}+V_{n} h$.

The first few hyperbolic generalized Pell numbers with positive subscript and negative subscript are given in the following Table 2.

\begin{tabular}{|c||c|c|}
\hline$n$ & $\widetilde{V}_{n}$ & $\widetilde{V}_{-n}$ \\
\hline \hline 0 & $V_{0}+h V_{1}$ & $\ldots$ \\
1 & $V_{1}+h\left(V_{0}+2 V_{1}\right)$ & $V_{1}-2 V_{0}+h V_{0}$ \\
2 & $V_{0}+2 V_{1}+h\left(2 V_{0}+5 V_{1}\right)$ & $5 V_{0}-2 V_{1}+h\left(-2 V_{0}+V_{1}\right)$ \\
3 & $2 V_{0}+5 V_{1}+h\left(5 V_{0}+12 V_{1}\right)$ & $5 V_{1}-12 V_{0}+h\left(5 V_{0}-2 V_{1}\right)$ \\
4 & $5 V_{0}+12 V_{1}+h\left(12 V_{0}+29 V_{1}\right)$ & $29 V_{0}-12 V_{1}+h\left(-12 V_{0}+5 V_{1}\right)$ \\
5 & $12 V_{0}+29 V_{1}+h\left(29 V_{0}+70 V_{1}\right)$ & $29 V_{1}-70 V_{0}+h\left(29 V_{0}-12 V_{1}\right)$ \\
\hline
\end{tabular}

Table 2. A few hyperbolic generalized Pell numbers

Note that

$$
\widetilde{V}_{0}=V_{0}+h V_{1}, \quad \widetilde{V}_{1}=V_{1}+h V_{2}=V_{1}+h\left(V_{0}+2 V_{1}\right) .
$$

For hyperbolic Pell numbers (taking $V_{n}=P_{n}, P_{0}=0, P_{1}=1$ ) we get

$$
\widetilde{P}_{0}=h, \quad \widetilde{P}_{1}=1+2 h,
$$

and for hyperbolic Pell-Lucas numbers (taking $V_{n}=Q_{n}, Q_{0}=2, Q_{1}=2$ ) we get

$$
\widetilde{Q}_{0}=2+2 h, \quad \widetilde{Q}_{1}=2+6 h .
$$

A few hyperbolic Pell numbers and hyperbolic Pell-Lucas numbers with positive subscript and negative subscript are given in the following Table 3 and Table 4.

\begin{tabular}{|c||c|c|}
\hline$n$ & $\widetilde{P}_{n}$ & $\widetilde{P}_{-n}$ \\
\hline \hline 0 & $h$ & $\cdots$ \\
1 & $1+2 h$ & 1 \\
2 & $2+5 h$ & $-2+h$ \\
3 & $5+12 h$ & $5-2 h$ \\
4 & $12+29 h$ & $-12+5 h$ \\
5 & $29+70 h$ & $29-12 h$ \\
\hline
\end{tabular}

Table 3. Hyperbolic Pell numbers

\begin{tabular}{|c||c|c|}
\hline$n$ & $\widetilde{Q}_{n}$ & $\widetilde{Q}_{-n}$ \\
\hline \hline 0 & $2+2 h$ & $\cdots$ \\
1 & $2+6 h$ & $-2+2 h$ \\
2 & $6+14 h$ & $6-2 h$ \\
3 & $14+34 h$ & $-14+6 h$ \\
4 & $34+82 h$ & $34-14 h$ \\
5 & $82+198 h$ & $-82+34 h$ \\
\hline
\end{tabular}

Table 4. Hyperbolic Pell-Lucas numbers 
Now, we will state Binet's formula for the hyperbolic generalized Pell numbers and in the rest of the paper, we fix the following notations:

$$
\widetilde{\alpha}=1+\alpha h, \quad \widetilde{\beta}=1+\beta h .
$$

Note that we have the following identities:

$$
\begin{aligned}
\widetilde{\alpha} & =1+\alpha h, \\
\widetilde{\beta} & =1+\beta h, \\
\widetilde{\alpha} \widetilde{\beta} & =2 h, \\
\widetilde{\alpha}^{2} & =2 \alpha+2+2 \alpha h, \\
\widetilde{\beta}^{2} & =2 \beta+2+2 \beta h, \\
\widetilde{\alpha}^{2} \widetilde{\beta} & =2 \alpha+2 h, \\
\widetilde{\alpha}^{2} & =2 \beta+2 h, \\
\widetilde{\alpha}^{2} \widetilde{\beta}^{2} & =4 .
\end{aligned}
$$

Theorem 1 (Binet's Formula). For any integer $n$, the $n$-th hyperbolic generalized Pell number is:

$$
\widetilde{V}_{n}=\frac{A \widetilde{\alpha} \alpha^{n}-B \widetilde{\beta} \beta^{n}}{\alpha-\beta} .
$$

Proof. Using Binet's formula

$$
V_{n}=\frac{A \alpha^{n}-B \beta^{n}}{\alpha-\beta}
$$

of the generalized Pell numbers, we obtain

$$
\widetilde{V}_{n}=V_{n}+h V_{n+1}=\frac{A \alpha^{n}-B \beta^{n}}{\alpha-\beta}+h \frac{A \alpha^{n+1}-B \beta^{n+1}}{\alpha-\beta}=\frac{A(1+\alpha h) \alpha^{n}-B(1+\beta h) \beta^{n}}{\alpha-\beta} .
$$

This proves (2.3).

As special cases, for any integer $n$, the Binet's Formula of $n$-th hyperbolic Pell number is

$$
\widetilde{P}_{n}=\frac{\widetilde{\alpha} \alpha^{n}-\widetilde{\beta} \beta^{n}}{\alpha-\beta}
$$

and the Binet's Formula of $n$-th hyperbolic Pell-Lucas number is

$$
\widetilde{Q}_{n}=\widetilde{\alpha} \alpha^{n}+\widetilde{\beta} \beta^{n}
$$

Next, we present generating function.

Theorem 2. The generating function for the hyperbolic generalized Pell numbers is:

$$
\sum_{n=0}^{\infty} \widetilde{V}_{n} x^{n}=\frac{\widetilde{V}_{0}+\left(\widetilde{V}_{1}-2 \widetilde{V}_{0}\right) x}{1-2 x-x^{2}} .
$$


Proof. Let

$$
g(x)=\sum_{n=0}^{\infty} \widetilde{V}_{n} x^{n}
$$

be the generating function of the hyperbolic generalized Pell numbers. Then, using the definition of the hyperbolic generalized Pell numbers, and substracting $2 x g(x)$ and $x^{2} g(x)$ from $g(x)$, we obtain (note the shift in the index $n$ in the third line)

$$
\begin{aligned}
\left(1-2 x-x^{2}\right) g(x) & =\sum_{n=0}^{\infty} \widetilde{V}_{n} x^{n}-2 x \sum_{n=0}^{\infty} \widetilde{V}_{n} x^{n}-x^{2} \sum_{n=0}^{\infty} \widetilde{V}_{n} x^{n} \\
& =\sum_{n=0}^{\infty} \widetilde{V}_{n} x^{n}-2 \sum_{n=0}^{\infty} \widetilde{V}_{n} x^{n+1}-\sum_{n=0}^{\infty} \widetilde{V}_{n} x^{n+2} \\
& =\sum_{n=0}^{\infty} \widetilde{V}_{n} x^{n}-2 \sum_{n=1}^{\infty} \widetilde{V}_{n-1} x^{n}-\sum_{n=2}^{\infty} \widetilde{V}_{n-2} x^{n} \\
& =\left(\widetilde{V}_{0}+\widetilde{V}_{1} x\right)-2 \widetilde{V}_{0} x+\sum_{n=2}^{\infty}\left(\widetilde{V}_{n}-2 \widetilde{V}_{n-1}-\widetilde{V}_{n-2}\right) x^{n} \\
& =\left(\widetilde{V}_{0}+\widetilde{V}_{1} x\right)-2 \widetilde{V}_{0} x=\widetilde{V}_{0}+\left(\widetilde{V}_{1}-2 \widetilde{V}_{0}\right) x .
\end{aligned}
$$

Note that we used the recurrence relation $\widetilde{V}_{n}=2 \widetilde{V}_{n-1}+\widetilde{V}_{n-2}$. Rearranging above equation, we get

$$
g(x)=\frac{\widetilde{V}_{0}+\left(\widetilde{V}_{1}-2 \widetilde{V}_{0}\right) x}{1-2 x-x^{2}} .
$$

As special cases, the generating functions for the hyperbolic Pell and hyperbolic Pell-Lucas numbers are, respectively,

$$
\sum_{n=0}^{\infty} \widetilde{P}_{n} x^{n}=\frac{h+x}{1-2 x-x^{2}}
$$

and

$$
\sum_{n=0}^{\infty} \widetilde{Q}_{n} x^{n}=\frac{(2+2 h)+(-2+2 h) x}{1-2 x-x^{2}} .
$$

\section{Obtaining Binet formula from generating function}

We next find Binet formula of hyperbolic generalized Pell number $\left\{\widetilde{V}_{n}\right\}$ by the use of generating function for $\widetilde{V}_{n}$.

Theorem 3 (Binet formula of hyperbolic generalized Pell numbers).

$$
\widetilde{V}_{n}=\frac{d_{1} \alpha^{n}}{(\alpha-\beta)}-\frac{d_{2} \beta^{n}}{(\alpha-\beta)}
$$

where

$$
d_{1}=\widetilde{V}_{0} \alpha+\left(\widetilde{V}_{1}-2 \widetilde{V}_{0}\right), \quad d_{2}=\widetilde{V}_{0} \beta+\left(\widetilde{V}_{1}-2 \widetilde{V}_{0}\right) .
$$

Proof. Let $h(x)=1-2 x-x^{2}$. Then for some $\alpha$ and $\beta$ we write $h(x)=(1-\alpha x)(1-\beta x)$, i.e.,

$$
1-2 x-x^{2}=(1-\alpha x)(1-\beta x)
$$


Hence $\frac{1}{\alpha}$ and $\frac{1}{\beta}$ are the roots of $h(x)$. This gives $\alpha$ and $\beta$ as the roots of

$$
h\left(\frac{1}{x}\right)=1-\frac{2}{x}-\frac{1}{x^{2}}=0 .
$$

This implies $x^{2}-2 x-1=0$. Now, by (2.6) and (3.2), it follows that

$$
\sum_{n=0}^{\infty} \widetilde{V}_{n} x^{n}=\frac{\widetilde{V}_{0}+\left(\widetilde{V}_{1}-2 \widetilde{V}_{0}\right) x}{(1-\alpha x)(1-\beta x)} .
$$

Then we write

$$
\frac{\widetilde{V}_{0}+\left(\widetilde{V}_{1}-2 \widetilde{V}_{0}\right) x}{(1-\alpha x)(1-\beta x)}=\frac{A_{1}}{(1-\alpha x)}+\frac{A_{2}}{(1-\beta x)}
$$

So $\widetilde{V}_{0}+\left(\widetilde{V}_{1}-2 \widetilde{V}_{0}\right) x=A_{1}(1-\beta x)+A_{2}(1-\alpha x)$.

If we consider $x=\frac{1}{\alpha}$, we get $\widetilde{V}_{0}+\left(\widetilde{V}_{1}-2 \widetilde{V}_{0}\right) \frac{1}{\alpha}=A_{1}\left(1-\beta \frac{1}{\alpha}\right)$. This gives

$$
A_{1}=\frac{\widetilde{V}_{0} \alpha+\left(\widetilde{V}_{1}-2 \widetilde{V}_{0}\right)}{(\alpha-\beta)}=\frac{d_{1}}{(\alpha-\beta)} .
$$

Similarly, we obtain $\widetilde{V}_{0}+\left(\widetilde{V}_{1}-2 \widetilde{V}_{0}\right) \frac{1}{\beta}=A_{2}\left(1-\alpha \frac{1}{\beta}\right) \Rightarrow \widetilde{V}_{0} \beta+\left(\widetilde{V}_{1}-2 \widetilde{V}_{0}\right)=A_{2}(\beta-\alpha)$ and so

$$
A_{2}=-\frac{\widetilde{V}_{0} \beta+\left(\widetilde{V}_{1}-2 \widetilde{V}_{0}\right)}{(\alpha-\beta)}=-\frac{d_{2}}{(\alpha-\beta)} .
$$

Thus (3.3) can be written as

$$
\sum_{n=0}^{\infty} \widetilde{V}_{n} x^{n}=A_{1}(1-\alpha x)^{-1}+A_{2}(1-\beta x)^{-1}
$$

This gives

$$
\sum_{n=0}^{\infty} \widetilde{V}_{n} x^{n}=A_{1} \sum_{n=0}^{\infty} \alpha^{n} x^{n}+A_{2} \sum_{n=0}^{\infty} \beta^{n} x^{n}=\sum_{n=0}^{\infty}\left(A_{1} \alpha^{n}+A_{2} \beta^{n}\right) x^{n} .
$$

Therefore, comparing coefficients on both sides of the above equality, we obtain $\widetilde{V}_{n}=A_{1} \alpha^{n}+A_{2} \beta^{n}$ and then we get (3.1).

Note that from (2.3) and (3.1) we have

$$
\begin{aligned}
& \left(V_{1}-V_{0} \beta\right) \widetilde{\alpha}=\widetilde{V}_{0} \alpha+\left(\widetilde{V}_{1}-2 \widetilde{V}_{0}\right), \\
& \left(V_{1}-V_{0} \alpha\right) \widetilde{\beta}=\widetilde{V}_{0} \beta+\left(\widetilde{V}_{1}-2 \widetilde{V}_{0}\right) .
\end{aligned}
$$

Next, using Theorem 3, we present the Binet formulas of hyperbolic Pell and hyperbolic Pell-Lucas numbers.

Corollary 4. Binet formulas of hyperbolic Pell and hyperbolic Pell-Lucas numbers are

$$
\widetilde{P}_{n}=\frac{\widetilde{\alpha} \alpha^{n}-\widetilde{\beta} \beta^{n}}{\alpha-\beta}, \quad \widetilde{Q}_{n}=\widetilde{\alpha} \alpha^{n}+\widetilde{\beta} \beta^{n},
$$

respectively. 


\section{Some identities}

We now present a few special identities for the hyperbolic generalized Pell sequence $\left\{\widetilde{V}_{n}\right\}$. The following theorem presents the Catalan's identity for the hyperbolic generalized Pell numbers.

Theorem 5 (Catalan's identity). For all integers $n$ and $m$, the following identity holds:

$$
\widetilde{V}_{n+m} \widetilde{V}_{n-m}-\widetilde{V}_{n}^{2}=\frac{(-1)^{n-m+1}\left((A+B) V_{2 m-1}+(A \beta+B \alpha) V_{2 m}-2(-1)^{m} A B\right)}{4} h .
$$

Proof. Using the Binet Formula

$$
\widetilde{V}_{n}=\frac{A \widetilde{\alpha} \alpha^{n}-B \widetilde{\beta} \beta^{n}}{\alpha-\beta}
$$

and

$$
A \alpha^{n}=\alpha V_{n}+V_{n-1}, \quad B \beta^{n}=\beta V_{n}+V_{n-1},
$$

we get

$$
\begin{aligned}
\widetilde{V}_{n+m} \widetilde{V}_{n-m}-\widetilde{V}_{n}^{2} & =\frac{\left(A \widetilde{\alpha} \alpha^{n+m}-B \widetilde{\beta} \beta^{n+m}\right)\left(A \widetilde{\alpha} \alpha^{n-m}-B \widetilde{\beta} \beta^{n-m}\right)-\left(A \widetilde{\alpha} \alpha^{n}-B \widetilde{\beta} \beta^{n}\right)^{2}}{(\alpha-\beta)^{2}} \\
& =\frac{(-1)^{n-m+1} A B\left(\alpha^{m}-\beta^{m}\right)^{2}}{8} 2 h \\
& =\frac{(-1)^{n-m+1}\left((A+B) V_{2 m-1}+(A \beta+B \alpha) V_{2 m}-2(-1)^{m} A B\right)}{4} h .
\end{aligned}
$$

As special cases of the above theorem, we give Catalan's identity of hyperbolic Pell and hyperbolic Pell-Lucas numbers. Firstly, we present Catalan's identity of hyperbolic Pell numbers.

Corollary 6 (Catalan's identity for the hyperbolic Pell numbers). For all integers $n$ and $m$, the following identity holds:

$$
\widetilde{P}_{n+m} \widetilde{P}_{n-m}-\widetilde{P}_{n}^{2}=\frac{(-1)^{n-m+1}\left(P_{2 m-1}+P_{2 m}-(-1)^{m}\right)}{2} h .
$$

Proof. Taking $V_{n}=P_{n}$ in Theorem 5 we get the required result.

Secondly, we give Catalan's identity of hyperbolic Pell-Lucas numbers.

Corollary 7 (Catalan's identity for the hyperbolic Pell-Lucas numbers). For all integers $n$ and $m$, the following identity holds: $\widetilde{Q}_{n+m} \widetilde{Q}_{n-m}-\widetilde{Q}_{n}^{2}=2(-1)^{n-m}\left(Q_{2 m}-2(-1)^{m}\right) h$.

Proof. Taking $V_{n}=Q_{n}$ in Theorem 5, we get the required result.

Note that for $m=1$ in Catalan's identity, we get the Cassini's identity for the hyperbolic generalized Pell sequence.

Corollary 8 (Cassini's identity). For all integers $n$, the following identity holds:

$$
\widetilde{V}_{n+1} \widetilde{V}_{n-1}-\widetilde{V}_{n}^{2}=\frac{(-1)^{n}\left((A+B) V_{1}+(A \beta+B \alpha) V_{2}+2 A B\right)}{4} h .
$$


As special cases of Cassini's identity, we give Cassini's identity of hyperbolic Pell and hyperbolic Pell-Lucas numbers. Firstly, we present Cassini's identity of hyperbolic Pell numbers.

Corollary 9 (Cassini's identity of hyperbolic Pell numbers). For all integers $n$, the following identity holds: $\widetilde{P}_{n+1} \widetilde{P}_{n-1}-\widetilde{P}_{n}^{2}=2(-1)^{n} h$.

Secondly, we give Cassini's identity of hyperbolic Pell-Lucas numbers.

Corollary 10 (Cassini's identity of hyperbolic Pell-Lucas numbers). For all integers $n$, the following identity holds: $\widetilde{Q}_{n+1} \widetilde{Q}_{n-1}-\widetilde{Q}_{n}^{2}=16(-1)^{n+1} h$.

The d'Ocagne's, Gelin-Cesàro's and Melham's identities can also be obtained by using the Binet Formula of the hyperbolic generalized Pell sequence:

$$
\widetilde{V}_{n}=\frac{A \widetilde{\alpha} \alpha^{n}-B \widetilde{\beta} \beta^{n}}{\alpha-\beta} .
$$

The next theorem presents d'Ocagne's, Gelin-Cesàro's and Melham's identities of the hyperbolic generalized Pell sequence $\left\{\widetilde{V}_{n}\right\}$.

Theorem 11. Let $n$ and $m$ be any integers. Then the following identities are true:

(a) (d'Ocagne's identity)

$$
\widetilde{V}_{m+1} \widetilde{V}_{n}-\widetilde{V}_{m} \widetilde{V}_{n+1}=2\left(V_{n} V_{m-1}-V_{m} V_{n-1}\right) h .
$$

(b) (Gelin-Cesàro's identity)

$$
\begin{aligned}
& \widetilde{V}_{n+2} \widetilde{V}_{n+1} \widetilde{V}_{n-1} \widetilde{V}_{n-2}-\widetilde{V}_{n}^{4}=\frac{A B(-1)^{n+1}}{2}\left(26(-1)^{n} A B+\left(6 V_{0}+18 V_{1}\right) V_{2 n}+\left(6 V_{0}+6 V_{1}\right) V_{2 n-1}+\right. \\
& \left.\left(\left(12 V_{0}+24 V_{1}\right) V_{2 n}+\left(3 A+3 B+\left(6 V_{0}+6 V_{1}\right)\right) V_{2 n-1}\right) h\right) .
\end{aligned}
$$

(c) (Melham's identity)

$$
\widetilde{V}_{n+1} \widetilde{V}_{n+2} \widetilde{V}_{n+6}-\widetilde{V}_{n+3}^{3}=2(-1)^{n} A B\left(\left(91 V_{n}+38 V_{n-1}\right)+\left(38 V_{n}+15 V_{n-1}\right) h\right) .
$$

Proof. (a) Using (1.7) and (1.8) we obtain

$$
\begin{aligned}
\widetilde{V}_{m+1} \widetilde{V}_{n}-\widetilde{V}_{m} \widetilde{V}_{n+1} & =\frac{A B \widetilde{\alpha} \widetilde{\beta}\left(-\alpha^{m+1} \beta^{n}-\alpha^{n} \beta^{m+1}+\alpha^{m} \beta^{n+1}+\alpha^{n+1} \beta^{m}\right)}{(\alpha-\beta)^{2}} \\
& =\frac{\left(\left(\alpha V_{n}+V_{n-1}\right)\left(\beta V_{m}+V_{m-1}\right)-\left(\alpha V_{m}+V_{m-1}\right)\left(\beta V_{n}+V_{n-1}\right)\right)}{(\alpha-\beta)} 2 h \\
& =2\left(V_{n} V_{m-1}-V_{m} V_{n-1}\right) h .
\end{aligned}
$$

(b) The proof is straightforward but lengthy, so we omit it.

(c) Using (1.7), (1.8) and Binet formula of $\widetilde{V}_{n}$ and the identities

$$
\widetilde{\alpha}^{2} \widetilde{\beta}=(2 \alpha+2 h), \quad \widetilde{\alpha} \widetilde{\beta}^{2}=(2 \beta+2 h),
$$

we obtain the required result. 
As special cases of the above theorem, we give the d'Ocagne's, Gelin-Cesàro's and Melham's identities of hyperbolic Pell and hyperbolic Pell-Lucas numbers. Firstly, we present the d'Ocagne's, Gelin-Cesàro's and Melham's identities of hyperbolic Pell numbers.

Corollary 12. Let $n$ and $m$ be any integers. Then, for the hyperbolic Pell numbers, the following identities are true:

(a) (d'Ocagne's identity)

$$
\widetilde{P}_{m+1} \widetilde{P}_{n}-\widetilde{P}_{m} \widetilde{P}_{n+1}=2\left(P_{n} P_{m-1}-P_{m} P_{n-1}\right) h .
$$

(b) (Gelin-Cesàro's identity)

$$
\begin{aligned}
& \widetilde{P}_{n+2} \widetilde{P}_{n+1} \widetilde{P}_{n-1} \widetilde{P}_{n-2}-\widetilde{P}_{n}^{4}=(-1)^{n+1}\left(13(-1)^{n}+\left(3 P_{0}+9 P_{1}\right) P_{2 n}+\left(3 P_{0}+3 P_{1}\right) P_{2 n-1}+\right. \\
& \left.\left(\left(6 P_{0}+12 P_{1}\right) P_{2 n}+\left(3+\left(3 P_{0}+3 P_{1}\right)\right) P_{2 n-1}\right) h\right) .
\end{aligned}
$$

(c) (Melham's identity)

$$
\widetilde{P}_{n+1} \widetilde{P}_{n+2} \widetilde{P}_{n+6}-\widetilde{P}_{n+3}^{3}=2(-1)^{n}\left(\left(91 P_{n}+38 P_{n-1}\right)+\left(38 P_{n}+15 P_{n-1}\right) h\right) .
$$

Secondly, we present the d'Ocagne's, Gelin-Cesàro's and Melham's identities of hyperbolic Pell-Lucas numbers.

Corollary 13. Let $n$ and $m$ be any integers. Then, for the hyperbolic Pell-Lucas numbers, the following identities are true:

(a) (d'Ocagne's identity)

$$
\widetilde{Q}_{m+1} \widetilde{Q}_{n}-\widetilde{Q}_{m} \widetilde{Q}_{n+1}=2\left(Q_{n} Q_{m-1}-Q_{m} Q_{n-1}\right) h .
$$

(b) (Gelin-Cesàro's identity)

$$
\begin{aligned}
& \widetilde{Q}_{n+2} \widetilde{Q}_{n+1} \widetilde{Q}_{n-1} \widetilde{Q}_{n-2}-\widetilde{Q}_{n}^{4}=8(-1)^{n}\left(104(-1)^{n+1}+\left(3 Q_{0}+9 Q_{1}\right) Q_{2 n}+\left(3 Q_{0}+3 Q_{1}\right) Q_{2 n-1}+\right. \\
& \left.\left(\left(6 Q_{0}+12 Q_{1}\right) Q_{2 n}+\left(3 Q_{0}+3 Q_{1}\right) Q_{2 n-1}\right) h\right) .
\end{aligned}
$$

(c) (Melham's identity)

$$
\widehat{Q}_{n+1} \widehat{Q}_{n+2} \widehat{Q}_{n+6}-\widehat{Q}_{n+3}^{3}=16(-1)^{n+1}\left(\left(91 Q_{n}+38 Q_{n-1}\right)+\left(38 Q_{n}+15 Q_{n-1}\right) h\right) .
$$

\section{Linear sums}

In this section, we give the summation formulas of the hyperbolic generalized Pell numbers with positive and negative subscripts. Now, we present the formula which give the summation formulas of the generalized Pell numbers.

Proposition 14. For the generalized Pell numbers, for $n \geq 0$ we have the following formulas:

(a) $\sum_{k=0}^{n} V_{k}=\frac{1}{2}\left(V_{n+2}-V_{n+1}-V_{1}+V_{0}\right)$.

(b) $\sum_{k=0}^{n} V_{2 k}=\frac{1}{2}\left(V_{2 n+1}-V_{1}+2 V_{0}\right)$.

(c) $\sum_{k=0}^{n} V_{2 k+1}=\frac{1}{2}\left(V_{2 n+2}-V_{2}+2 V_{1}\right)$.

Proof. For the proof, see Soykan [31] . 
Next, we present the formulas which give the summation of the first $n$ hyperbolic generalized Pell numbers.

Theorem 15. For $n \geq 0$, hyperbolic generalized Pell numbers have the following formulas:

(a) $\sum_{k=0}^{n} \widetilde{V}_{k}=\frac{1}{2}\left(\widetilde{V}_{n+2}-\widetilde{V}_{n+1}-\widetilde{V}_{1}+\widetilde{V}_{0}\right)$.

(b) $\sum_{k=0}^{n} \widetilde{V}_{2 k}=\frac{1}{2}\left(\widetilde{V}_{2 n+1}-\widetilde{V}_{1}+2 \widetilde{V}_{0}\right)$.

(c) $\sum_{k=0}^{n} \widetilde{V}_{2 k+1}=\frac{1}{2}\left(\widetilde{V}_{2 n+2}-\widetilde{V}_{0}\right)$.

Proof. (a) Note that using Proposition 14 (a) we get

$$
\begin{aligned}
\sum_{k=0}^{n} V_{k} & =\frac{1}{2}\left(V_{n+2}-V_{n+1}-V_{1}+V_{0}\right), \\
\sum_{k=0}^{n} V_{k+1} & =\frac{1}{2}\left(V_{n+3}-V_{n+2}-V_{1}-V_{0}\right) .
\end{aligned}
$$

Then it follows that

$$
\begin{aligned}
\sum_{k=0}^{n} \widetilde{V}_{k} & =\frac{1}{2}\left(V_{n+2}-V_{n+1}-V_{1}+V_{0}\right)+h \frac{1}{2}\left(V_{n+3}-V_{n+2}-V_{1}-V_{0}\right) \\
& =\frac{1}{2}\left(\left(V_{n+2}+j V_{n+3}\right)-\left(V_{n+1}+h V_{n+2}\right)+\left(-V_{1}+V_{0}\right)+h\left(-V_{1}-V_{0}\right)\right) \\
& =\frac{1}{2}\left(\widetilde{V}_{n+2}-\widetilde{V}_{n+1}+\left(\left(-V_{1}+V_{0}\right)+h\left(-V_{2}+V_{1}\right)\right)\right. \\
& \left.=\frac{1}{2}\left(\widetilde{V}_{n+2}-\widetilde{V}_{n+1}-\left(V_{1}+h V_{2}\right)\right)+\left(V_{0}+h V_{1}\right)\right) \\
& \left.=\frac{1}{2}\left(\widetilde{V}_{n+2}-\widetilde{V}_{n+1}-\left(V_{1}+h V_{2}\right)\right)+\left(V_{0}+h V_{1}\right)\right) \\
& =\frac{1}{2}\left(\widetilde{V}_{n+2}-\widetilde{V}_{n+1}-\widetilde{V}_{1}+\widetilde{V}_{0}\right) .
\end{aligned}
$$

This proves (a).

(b) Note that using Proposition 14 (b) and (c) we get

$$
\begin{aligned}
\sum_{k=0}^{n} V_{2 k} & =\frac{1}{2}\left(V_{2 n+1}-V_{1}+2 V_{0}\right), \\
\sum_{k=0}^{n} V_{2 k+1} & =\frac{1}{2}\left(V_{2 n+2}-V_{0}\right) .
\end{aligned}
$$

Then it follows that

$$
\begin{aligned}
\sum_{k=0}^{n} \widetilde{V}_{2 k} & =\frac{1}{2}\left(V_{2 n+1}-V_{1}+2 V_{0}\right)+h \frac{1}{2}\left(V_{2 n+2}-V_{0}\right) \\
& =\frac{1}{2}\left(\left(V_{2 n+1}+h V_{2 n+2}\right)+\left(\left(-V_{1}+2 V_{0}\right)+h\left(-V_{0}\right)\right)\right) \\
& =\frac{1}{2}\left(\left(V_{2 n+1}+h V_{2 n+2}\right)+\left(\left(-V_{1}+2 V_{0}\right)+h\left(-V_{2}+2 V_{1}\right)\right)\right. \\
& =\frac{1}{2}\left(\left(V_{2 n+1}+h V_{2 n+2}\right)-\left(V_{1}+h V_{2}\right)+2\left(V_{0}+h V_{1}\right)\right) \\
& =\frac{1}{2}\left(\widetilde{V}_{2 n+1}-\widetilde{V}_{1}+2 \widetilde{V}_{0}\right) .
\end{aligned}
$$


(c) Note that using Proposition 14 (b) and (c) we get

$$
\sum_{k=0}^{n} V_{2 k+2}=\frac{1}{2}\left(V_{2 n+3}-V_{1}\right) \text {. }
$$

Then it follows that

$$
\begin{aligned}
\sum_{k=0}^{n} \widetilde{V}_{2 k+1} & =\frac{1}{2}\left(V_{2 n+2}-V_{0}\right)+h \frac{1}{2}\left(V_{2 n+3}-V_{1}\right) \\
& =\frac{1}{2}\left(\left(V_{2 n+2}+h V_{2 n+3}\right)-\left(V_{0}+h V_{1}\right)\right) \\
& =\frac{1}{2}\left(\widetilde{V}_{2 n+2}-\left(V_{0}+h V_{1}\right)\right) \\
& =\frac{1}{2}\left(\widetilde{V}_{2 n+2}-\widetilde{V}_{0}\right) .
\end{aligned}
$$

This completes the proof.

As a first special case of the above theorem, we have the following summation formulas for hyperbolic Pell numbers:

Corollary 16. For $n \geq 0$, hyperbolic Pell numbers have the following properties:

(a) $\sum_{k=0}^{n} \widetilde{P}_{k}=\frac{1}{2}\left(\widetilde{P}_{n+2}-\widetilde{P}_{n+1}-\widetilde{P}_{1}+\widetilde{P}_{0}\right)=\frac{1}{2}\left(\widetilde{P}_{n+2}-\widetilde{P}_{n+1}-(1+h)\right)$.

(b) $\sum_{k=0}^{n} \widetilde{P}_{2 k}=\frac{1}{2}\left(\widetilde{P}_{2 n+1}-\widetilde{P}_{1}+2 \widetilde{P}_{0}\right)=\frac{1}{2}\left(\widetilde{P}_{2 n+1}-1\right)$.

(c) $\sum_{k=0}^{n} \widetilde{P}_{2 k+1}=\frac{1}{2}\left(\widetilde{P}_{2 n+2}-\widetilde{P}_{0}\right)=\frac{1}{2}\left(\widetilde{P}_{2 n+2}-h\right)$.:

As a second special case of the above theorem, we have the following summation formulas for hyperbolic Pell-Lucas numbers:

Corollary 17. For $n \geq 0$, hyperbolic Pell-Lucas numbers have the following properties.

(a) $\sum_{k=0}^{n} \widetilde{Q}_{k}=\frac{1}{2}\left(\widetilde{Q}_{n+2}-\widetilde{Q}_{n+1}-\widetilde{Q}_{1}+\widetilde{Q}_{0}\right)=\frac{1}{2}\left(\widetilde{Q}_{n+2}-\widetilde{Q}_{n+1}-4 h\right)$.

(b) $\sum_{k=0}^{n} \widetilde{Q}_{2 k}=\frac{1}{2}\left(\widetilde{Q}_{2 n+1}-\widetilde{Q}_{1}+2 \widetilde{Q}_{0}\right)=\frac{1}{2}\left(\widetilde{Q}_{2 n+1}+2-2 h\right)$.

(c) $\sum_{k=0}^{n} \widetilde{Q}_{2 k+1}=\frac{1}{2}\left(\widetilde{Q}_{2 n+2}-\widetilde{Q}_{0}\right)=\frac{1}{2}\left(\widetilde{Q}_{2 n+2}-(2+2 h)\right)$.

Now, we present the formula which give the summation formulas of the generalized Pell numbers with negative subscripts.

Proposition 18. For $n \geq 1$ we have the following formulas:

(a) $\sum_{k=1}^{n} V_{-k}=\frac{1}{2}\left(-3 V_{-n-1}-V_{-n-2}+V_{1}-V_{0}\right)$.

(b) $\sum_{k=1}^{n} V_{-2 k}=\frac{1}{2}\left(-V_{-2 n-1}+V_{1}-2 V_{0}\right)$.

(c) $\sum_{k=1}^{n} V_{-2 k+1}=\frac{1}{2}\left(-V_{-2 n}+V_{0}\right)$.

Proof. This is given in Soykan [31]. 
Next, we present the formulas which give the summation of the first $n$ hyperbolic generalized Pell numbers with negative subscripts

Theorem 19. For $n \geq 1$, hyperbolic generalized Pell numbers have the following formulas:

(a) $\sum_{k=1}^{n} \widetilde{V}_{-k}=\frac{1}{2}\left(-3 \widetilde{V}_{-n-1}-\widetilde{V}_{-n-2}+\widetilde{V}_{1}-\widetilde{V}_{0}\right)$.

(b) $\sum_{k=1}^{n} \widetilde{V}_{-2 k}=\frac{1}{2}\left(-\widetilde{V}_{-2 n-1}+\widetilde{V}_{1}-2 \widetilde{V}_{0}\right)$.

(c) $\sum_{k=1}^{n} \widetilde{V}_{-2 k+1}=\frac{1}{2}\left(-\widetilde{V}_{-2 n}+\widetilde{V}_{0}\right)$.

Proof. We prove (a). Note that using Proposition 14 (a) we get

$$
\begin{aligned}
\sum_{k=1}^{n} V_{-k} & =\frac{1}{2}\left(-3 V_{-n-1}-V_{-n-2}+V_{1}-V_{0}\right), \\
\sum_{k=1}^{n} V_{-k+1} & =\frac{1}{2}\left(-3 V_{-n}-V_{-n-1}+V_{1}+V_{0}\right) .
\end{aligned}
$$

Then it follows that

$$
\begin{aligned}
\sum_{k=1}^{n} \widetilde{V}_{-k} & =\frac{1}{2}\left(-3 V_{-n-1}-V_{-n-2}+V_{1}-V_{0}\right)+j \frac{1}{2}\left(-3 V_{-n}-V_{-n-1}+V_{1}+V_{0}\right) \\
& =\frac{1}{2}\left(3\left(V_{-n-1}+j V_{-n}\right)-\left(V_{-n-2}+j V_{-n-1}\right)+\left(V_{1}-V_{0}\right)+j\left(V_{1}+V_{0}\right)\right) \\
& =\frac{1}{2}\left(-3 \widetilde{V}_{-n-1}-\widetilde{V}_{-n-2}+\left(\left(V_{1}-V_{0}\right)+j\left(V_{2}-V_{1}\right)\right)\right. \\
& \left.=\frac{1}{2}\left(-3 \widetilde{V}_{-n-1}-\widetilde{V}_{-n-2}+\left(V_{1}+j V_{2}\right)+\widetilde{V}_{0}\right)-\left(V_{0}+j V_{1}\right)\right) \\
& =\frac{1}{2}\left(-3 \widetilde{V}_{-n-1}-\widetilde{V}_{-n-2}+\widetilde{V}_{1}-\widetilde{V}_{0}\right)
\end{aligned}
$$

This proves (a). Formulas (b) and (c) can be proved similarly.

As a first special case of above theorem, we have the following summation formulas for hyperbolic Pell numbers:

Corollary 20. For $n \geq 1$, hyperbolic Pell numbers have the following properties:

(a) $\sum_{k=1}^{n} \widetilde{P}_{-k}=\frac{1}{2}\left(-3 \widetilde{P}_{-n-1}-\widetilde{P}_{-n-2}+\widetilde{P}_{1}-\widetilde{P}_{0}\right)=\frac{1}{2}\left(-3 \widetilde{P}_{-n-1}-\widetilde{P}_{-n-2}+(1+h)\right)$.

(b) $\sum_{k=1}^{n} \widetilde{P}_{-2 k}=\frac{1}{2}\left(-\widetilde{P}_{-2 n-1}+\widetilde{P}_{1}-2 \widetilde{P}_{0}\right)=\frac{1}{2}\left(-\widetilde{P}_{-2 n-1}+1\right)$.

(c) $\sum_{k=1}^{n} \widetilde{P}_{-2 k+1}=\frac{1}{2}\left(-\widetilde{P}_{-2 n}+\widetilde{P}_{0}\right)=\frac{1}{2}\left(-\widetilde{P}_{-2 n}+h\right)$.

As a second special case of above theorem, we have the following summation formulas for hyperbolic Pell-Lucas numbers:

Corollary 21. For $n \geq 1$, hyperbolic Pell-Lucas numbers have the following properties.

(a) $\sum_{k=1}^{n} \widetilde{Q}_{-k}=\frac{1}{2}\left(-3 \widetilde{Q}_{-n-1}-\widetilde{Q}_{-n-2}+\widetilde{Q}_{1}-\widetilde{Q}_{0}\right)=\frac{1}{2}\left(-3 \widetilde{Q}_{-n-1}-\widetilde{Q}_{-n-2}+4 h\right)$.

(b) $\sum_{k=1}^{n} \widetilde{Q}_{-2 k}=\frac{1}{2}\left(-\widetilde{Q}_{-2 n-1}+\widetilde{Q}_{1}-2 \widetilde{Q}_{0}\right)=\frac{1}{2}\left(-\widetilde{Q}_{-2 n-1}+(-2+2 h)\right)$.

(c) $\sum_{k=1}^{n} \widetilde{Q}_{-2 k+1}=\frac{1}{2}\left(-\widetilde{Q}_{-2 n}+\widetilde{Q}_{0}\right)=\frac{1}{2}\left(-\widetilde{Q}_{-2 n}+(2+2 h)\right)$. 


\section{Matrices related to hyperbolic generalized Pell numbers}

We define the square matrix $N$ of order 2 as:

$$
N=\left(\begin{array}{ll}
2 & 1 \\
1 & 0
\end{array}\right)
$$

such that $\operatorname{det} N=-1$. Induction proof may be used to establish

$$
N^{n}=\left(\begin{array}{cc}
P_{n+1} & P_{n} \\
P_{n} & P_{n-1}
\end{array}\right)
$$

and (the matrix formulation of $V_{n}$ )

$$
\left(\begin{array}{c}
V_{n+1} \\
V_{n}
\end{array}\right)=\left(\begin{array}{ll}
2 & 1 \\
1 & 0
\end{array}\right)^{n}\left(\begin{array}{l}
V_{1} \\
V_{0}
\end{array}\right) .
$$

Now, we define the matrices $N_{V}$ as

$$
N_{V}=\left(\begin{array}{cc}
\widetilde{V}_{3} & \widetilde{V}_{2} \\
\widetilde{V}_{2} & \widetilde{V}_{1}
\end{array}\right)
$$

This matrice $N_{V}$ is called hyperbolic generalized Pell matrix. As special cases, hyperbolic Pell matrix and hyperbolic Pell-Lucas matrix are, respectively,

$$
N_{P}=\left(\begin{array}{cc}
\widetilde{P}_{3} & \widetilde{P}_{2} \\
\widetilde{P}_{2} & \widetilde{P}_{1}
\end{array}\right), \quad N_{Q}=\left(\begin{array}{cc}
\widetilde{Q}_{3} & \widetilde{Q}_{2} \\
\widetilde{Q}_{2} & \widetilde{Q}_{1}
\end{array}\right),
$$

respectively.

Theorem 22. For $n \geq 0$, the following is valid:

$$
N_{V}\left(\begin{array}{ll}
2 & 1 \\
1 & 0
\end{array}\right)^{n}=\left(\begin{array}{cc}
\widetilde{V}_{n+3} & \widetilde{V}_{n+2} \\
\widetilde{V}_{n+2} & \widetilde{V}_{n+1}
\end{array}\right) .
$$

Proof. We prove by mathematical induction on $n$. If $n=0$, then the result is clear. Now, we assume it is true for $n=k$, that is

$$
N_{V} N^{k}=\left(\begin{array}{cc}
\widetilde{V}_{k+3} & \widetilde{V}_{k+2} \\
\widetilde{V}_{k+2} & \widetilde{V}_{k+1}
\end{array}\right)
$$

If we use (2.1), then we have $\widetilde{V}_{k+2}=2 \widetilde{V}_{k+1}+\widetilde{V}_{k}$. Then, by induction hypothesis, we obtain

$$
\begin{aligned}
N_{V} N^{k+1} & =\left(N_{V} N^{k}\right) N=\left(\begin{array}{cc}
\widetilde{V}_{k+3} & \widetilde{V}_{k+2} \\
\widetilde{V}_{k+2} & \widetilde{V}_{k+1}
\end{array}\right)\left(\begin{array}{ll}
2 & 1 \\
1 & 0
\end{array}\right)=\left(\begin{array}{cc}
2 \widetilde{V}_{k+3}+\widetilde{V}_{k+2} & \widetilde{V}_{k+3} \\
2 \widetilde{V}_{k+2}+\widetilde{V}_{k+1} & \widetilde{V}_{k+2}
\end{array}\right) \\
& =\left(\begin{array}{cc}
\widetilde{V}_{k+4} & \widetilde{V}_{k+3} \\
\widetilde{V}_{k+3} & \widetilde{V}_{k+2}
\end{array}\right) .
\end{aligned}
$$

Thus, (6.3) holds for all non-negative integers $n$. 
Remark 23. The above theorem is true for $n \leq-1$. It can also be proved by induction.

Corollary 24. For all integers $n$, the following holds: $\widetilde{V}_{n+2}=\widetilde{V}_{2} P_{n+1}+\widetilde{V}_{1} P_{n}$.

Proof. The proof can be seen by the coefficient of the matrix $N_{V}$ and (6.1).

Taking $V_{n}=P_{n}$ and $V_{n}=Q_{n}$, respectively, in the above corollary, we obtain the following results.

Corollary 25. For all integers $n$, the followings are true.

(a) $\widetilde{P}_{n+2}=\widetilde{P}_{2} P_{n+1}+\widetilde{P}_{1} P_{n}$.

(b) $\widetilde{Q}_{n+2}=\widetilde{Q}_{2} P_{n+1}+\widetilde{Q}_{1} P_{n}$.

Remark 26. As a further study, the results of this paper can be extended to the work in [26].

\section{References}

[1] Akar, M., Yüce, S., \& Şahin, Ş. (2018). On the Dual Hyperbolic Numbers and the Complex Hyperbolic Numbers, Journal of Computer Science \& Computational Mathematics, 8(1), $1-6$.

[2] Baez, J. (2002). The octonions, Bull. Amer. Math. Soc., 39(2), 145-205.

[3] Bicknell, M. (1975). A primer on the Pell sequence and related sequence, The Fibonacci Quarterly, 13(4), 345-349.

[4] Biss, D.K., Dugger, D., \& Isaksen, D.C. (2008). Large annihilators in Cayley-Dickson algebras, Communication in Algebra, 36(2), 632-664.

[5] Cheng, H. H., \& Thompson, S. (1996). Dual Polynomials and Complex Dual Numbers for Analysis of Spatial Mechanisms, Proc. of ASME 24th Biennial Mechanisms Conference, Irvine, CA, 19-22 August, 1996.

[6] Cockle, J. (1849). On a New Imaginary in Algebra, The London, Edinburgh, and Dublin Philosophical Magazine and Journal of Science, 3(34), 37-47.

[7] Dasdemir, A. (2011). On the Pell, Pell-Lucas and Modified Pell Numbers By Matrix Method, Applied Mathematical Sciences, 5(64), 3173-3181.

[8] Dikmen, C. M. (2019). Hyperbolic Jacobsthal Numbers, Asian Research Journal of Mathematics, 15(4), 1-9.

[9] Ercolano, J. (1979). Matrix generator of Pell sequence, Fibonacci Quarterly, 17(1), 71-77.

[10] Fjelstad, P., \& G. Gal Sorin. (1998). n-dimensional Hyperbolic Complex Numbers, Advances in Applied Clifford Algebras, 8(1), 47-68. 
[11] Gökbas, H., \& Köse, H. (2017). Some sum formulas for products of Pell and Pell-Lucas numbers, Int. J. Adv. Appl. Math. and Mech., 4(4), 1-4.

[12] Hamilton, W. R. (1969). Elements of Quaternions, Chelsea Publishing Company, New York.

[13] Horadam, A. F. (1971). Pell identities, Fibonacci Quarterly, 9 (3), 245-263.

[14] Imaeda, K., \& Imaeda, M. (2000). Sedenions: algebra and analysis, Applied Mathematics and Computation, 115, 77-88.

[15] Jancewicz, B. (1996). The extended Grassmann algebra of $R^{3}$, in Clifford (Geometric) Algebras with Applications and Engineering, Birkhauser, Boston, 389-421.

[16] Kantor, I., \& Solodovnikov, A. (1989). Hypercomplex Numbers, Springer-Verlag, New York.

[17] Kiliç, E., \& Taşçi, D. (2005). The Linear Algebra of The Pell Matrix, Boletín de la Sociedad Matemática Mexicana, 11(2), 163-174.

[18] Kiliç, E., \& Taşçi, D. (2006). The Generalized Binet Formula, Representation and Sums of the Generalized Order- $k$ Pell Numbers, Taiwanese Journal of Mathematics, 10(6), 1661-1670.

[19] Kiliç, E., \& Stanica, P. (2011). A Matrix Approach for General Higher Order Linear Recurrences, Bulletin of the Malaysian Mathematical Sciences Society, 34(1), 51-67.

[20] Khrennikov, A., \& Segre, G. (2005). An Introduction to Hyperbolic Analysis. Available online: http: //arxiv.org/abs/math-ph/0507053v2.

[21] Koshy, T. (2014). Pell and Pell-Lucas Numbers with Applications, Springer, New York.

[22] Kravchenko, V. V. (2009). Hyperbolic Numbers and Analytic Functions. In: Applied Pseudoanalytic Function Theory. Frontiers in Mathematics. Birkhäuser Basel, DOI: https://doi.org/10.1007/978-3-0346-0004-0_11.

[23] Melham, R. (1999). Sums Involving Fibonacci and Pell Numbers, Portugaliae Mathematica, 56(3), 309-317.

[24] Moreno, G. (1998). The zero divisors of the Cayley-Dickson algebras over the real numbers, Bol. Soc. Mat. Mexicana (3), 4(1), 13-28.

[25] Motter A. E., \& Rosa, A. F. (1998). Hyperbolic calculus, Adv. Appl. Clifford Algebra, 8(1), $109-128$.

[26] Ollerton, R. L. \& Shannon, A. G. (1992). An extension of circular and hyperbolic functions, International Journal of Mathematical Education in Science and Technology, 23(4), 611-620. 
[27] Sloane, N.J.A., The on-line encyclopedia of integer sequences. Available online: http: //oeis.org/.

[28] Sobczyk, G. (1995). The Hyperbolic Number Plane, The College Mathematics Journal, 26(4), 268-280.

[29] Sobczyk, G. (2013). Complex and Hyperbolic Numbers. In: New Foundations in Mathematics. Birkhäuser, Boston. DOI 10.1007/978-0-8176-8385-6_2.

[30] Soykan, Y. (2019). Tribonacci and Tribonacci-Lucas Sedenions. Mathematics, 7(1), 74.

[31] Soykan, Y. (2019). On Summing Formulas For Generalized Fibonacci and Gaussian Generalized Fibonacci Numbers, Advances in Research, 20(2), 1-15.

[32] Soykan, Y. (2019). On Generalized Third-Order Pell Numbers, Asian Journal of Advanced Research and Reports, 6(1), 1-18.

[33] Soykan, Y. (2019). A Study of Generalized Fourth-Order Pell Sequences, Journal of Scientific Research and Reports, 25(1-2), 1-18.

[34] Soykan, Y. (2019). Properties of Generalized Fifth-Order Pell Numbers, Asian Research Journal of Mathematics, 15(3), 1-18.

[35] Soykan, Y. (2019). On Hyperbolic Numbers With Generalized Fibonacci Numbers Components, Researchgate Preprint, DOI: 10.13140/RG.2.2.19903.87207.

[36] Torunbalcı Aydın, F. (2019). Hyperbolic Fibonacci Sequence, Universal Journal of Mathematics and Applications, 2(2), 59-64. 